\title{
Validité externe et méthode expérimentale
}

External Validity and experimental Method

\section{Thierry Meyer}

\section{(2) OpenEdition}

\section{Journals}

Édition électronique

URL : http://journals.openedition.org/questionsdecommunication/4655

DOI : 10.4000/questionsdecommunication.4655

ISSN : 2259-8901

\section{Éditeur}

Presses universitaires de Lorraine

\section{Édition imprimée}

Date de publication : 30 juin 2005

Pagination : 209-222

ISBN : 978-2-86480-859-6

ISSN : 1633-5961

\section{Référence électronique}

Thierry Meyer, « Validité externe et méthode expérimentale », Questions de communication [En ligne], 7| 2005, mis en ligne le 10 mai 2012, consulté le 10 décembre 2020. URL : http://

journals.openedition.org/questionsdecommunication/4655; DOI : https://doi.org/10.4000/ questionsdecommunication.4655 


\section{$>$ ÉCHANGES}

THIERRY MEYER

Processus cognitifs et conduites interactives

Université Paris 10

thierry-marcel.meyer@u-paris10.fr

\section{VAIDITÉ EXTERNE ET MÉTHO DE EXPÉRIMEN TAIE}

Résumé. - La méthode expérimentale en psychologie est souvent questionnée sous l'angle de la généralisation de ses résultats et de ses modèles (validité externe). Les critères classiques de la validité externe sont exposés. L'accent est mis sur la distinction entre validité externe fondée sur les situations expérimentales et celle fondée sur les processus postulés par un modèle. Les conséquences pour un dialogue entre les disciplines et les méthodes dans le champ de l'information et de la communication sont discutées.

Mots clés. - Méthode expérimentale, validité externe, psychologie. 
a livraison de Questions de communication consacrée au thème

"Psychologie sociale, traitements et effets des médias " (Chabrol,

Courbet, Fourquet-Courbet, 2004) suscite une occasion de réflexion sur la méthode expérimentale. Peu fréquentée dans le champ académique de l'information et de la communication, l'expérimentation reste pour la psychologie le moyen privilégié de légitimation des recherches (Breckler, 2004). De fait, toutes les contributions recourent à cette méthode, soit pour présenter des résultats originaux, soit pour proposer des synthèses quantitatives (méta-analyses) elles-mêmes fondées sur des approches expérimentales (effet de l' " appel à la peur » dans la communication sur les risques, ou effet de la violence rencontrée dans les médias sur les comportements agressifs). L'essentiel des articles porte sur les effets attendus de la présentation d'un message (publicité commerciale, santé publique), d'une séquence filmée, d'un contexte psychosocial de communication dont on a expérimentalement isolé une propriété ou un ensemble de propriétés. La critique la plus courante à l'exposé de travaux expérimentaux, et spécialement dans le domaine de l'information et de la communication, est que la méthode expérimentale produit des artefacts qui ne rendent pas compte des pratiques et des processus "réels". A priori, les recherches expérimentales souffriraient d'un déficit de généralisation ou encore de validité " externe » pour employer le jargon expérimental lui-même.

La préoccupation de validé externe est présente chez les auteurs de ce numéro, en particulier sous l'angle de la validité écologique, c'est-à-dire le fait que les participants de leurs recherches sont exposés à des situations et des messages qu'ils ont quelques chances de rencontrer en dehors du cadre expérimental. Ainsi Didier Courbet, dans son investigation sur la publicité sur l'internet, fabrique un site « ressemblant " à ceux que les participants pourraient rencontrer en naviguant sur le net. Claude Chabrol et Gaëlle Diligaert (messages de prévention en sécurité routière, - 2004) comme Miruna Radu (publicité pour produits cosmétiques, - 2004) veillent soigneusement à la crédibilité de leurs messages, quand bien même construisent-ils ceux-ci de toute pièce. $D$ ans leur méta-analyse, qui intègre des dizaines de recherches sur les effets des messages fondés sur l'appel à la peur dans la santé publique, Mike Allen et Kim W itte (2004) constatent que les messages mis à l'étude sont, pour une grande part, " artificiels" dans le sens qu'ils sont conçus pour les besoins d'une recherche. La valeur du modèle à propos de campagnes en grandeur réelle reste encore à entreprendre.

Dans quelle mesure les modèles mis à l'épreuve dans une situation expérimentale, nécessairement réductrice, peuvent-ils être généralisés ? Mon propos principal porte sur la validité « externe ", telle qu'elle est conceptualisée dans le cadre même de la psychologie expérimentale et 
selon les termes de ceux qui pratiquent les méthodes expérimentales. Mon point de vue n'est pas celui de l'épistémologue, mais celui d'un chercheur impliqué dans des programmes expérimentaux et qui doit rendre des comptes de leur portée au-delà du cadre expérimental. Après un rappel, intentionnellement didactique, des bases d'une méthode expérimentale, je développerai un cadre systématique pour l'appréciation de la validité externe. La question de la validité externe est une étape indispensable et pragmatique pour un dialogue interdisciplinaire dans le champ de l'information et de la communication qui, nécessairement, confronte les options méthodologiques.

\section{Métho de expérimentale : \\ réductio nnisme raiso nné et maximisatio $n$ de la validité interne}

L'expérimentation repose sur un réductionnisme raisonné. Sur la base d'un nombre limité de variables, un modèle est proposé qui explicite les liens, et spécialement ceux de cause à effet entre ces variables. Une situation expérimentale est construite afin de tester une hypothèse issue d'un modèle (voir figure 1 : Deux niveaux d'appréciation de la validité externe). L'objectif d'une approche expérimentale est de rendre plus plausibles les conclusions issues des observations en vue de procéder à une inférence causale.Traditionnellement, en psychologie, on se pose la question de savoir si un " traitement " particulier a un effet sur une mesure recueillie auprès de participants qui se prêtent à une recherche. Prenons le cas où l'intérêt du chercheur porte sur une seule variable. Admettons, par exemple, que l'objet d'intérêt soit le rôle de la familiarité de la source sur la persuasion provenant de l'exposition à un message. Une source familière rend-elle un message plus attractif ? II s'agit de limiter autant que possible la confusion entre cette variable d'intérêt et d'autres variables qui pourraient être mélangées ou confondues avec celle-ci. Ainsi Max W eisbuch, Diane Mackie et Teresa Garcia-Marques (2003) présentent-ils une recherche où la familiarité de la source est manipulée, dans un premier temps, par l'exposition préalable à un visage selon une durée telle que cette exposition n'est pas perceptible par les participants (4 fois pendant 23 millisecondes). $D$ ans un second temps, ce visage est présenté comme la source d'un message sur un sujet controversé (introduction de taxes à l'entrée de routes). Personne ne doute que, pour décider si l'exposition préalable au visage a un effet sur la réception du message, il faut comparer un groupe qui a été exposé et un groupe qui ne l'a pas été. La méthode expérimentale stricto sensu recourt à l'échantillonnage aléatoire :le traitement est affecté au hasard 
à des participants (randomisation). Le but est d'opérer des comparaisons selon un raisonnement caeteris paribus ou " toutes choses égales par ailleurs ". Les plans d'expérience sont un instrument pragmatique pour éviter de confondre deux variables concomitantes (Aronson, W ilson, Brewer, 1998). En passant, rappelons que la comparaison de deux groupes naturels (hommes vs femmes, spectateurs assidus vs occasionnels, fumeurs vs non-fumeurs, etc.) ne permet pas les mêmes inférences causales que la méthode expérimentale (approche corrélationnelle, dite aussi quasiexpérimentale).

Pour reprendre les termes de Donald Campbell (1957), la planification des observations répond d'abord à des préoccupations de validité " interne "'.Q uel que soit le raffinement du plan, la validité interne n'est jamais assurée. Le plus souvent, est examinée l'adéquation entre le plan de recherche et les objectifs de recherche. $\downarrow$ ne passerai pas en revue toutes les " menaces » envers la validité interne qui s'appliquent, plus ou moins, non seulement selon le plan choisi, mais aussi selon le patron des résultats (Brewer, 2000 ; D elhomme, Meyer, 2002).

\section{Examiner la validité externe}

La validité externe porte sur la possibilité de généraliser les résultats observés au-delà de la situation expérimentale elle-même. Alors que la validité interne est discutée sur la base de critères logiques (plans d'expérience) et de mesures, la validité externe est souvent abordée de manière plus qualitative.

\section{Les critères élémentaires : UTO S}

Sur quels critères la validité externe s'appuie-t-elle ? Elle se décline selon quatre dimensions : les unités, traitements, observations et sites, résumé par l'acronyme UTOS, pour reprendre la proposition de Lee Cronbach (1982).

\footnotetext{
${ }^{1}$ « N ous distinguerons deux types principaux de validité. Premièrement la validité interne ;la validité interne représente l'exigence minimale ;quand on en parle,c'est pour demander si la stimulation expérimentale a bien déterminé des différences dans la situation singulière de telle expérience. Deuxièmement, il y a la validité externe, la représentativité, la généralisabilité des résultats de l'expérience ; quand on en parle c'est pour demander à quelles populations, à quels contextes, à quelles variables l'effet enregistré peut être étendu » (Campbell, $1957: 47$ ).
} 
En général, les unités sont les individus qui participent aux recherches. La plupart des recherches expérimentales recourent à des échantillons de taille insuffisante pour prétendre à la représentativité de la population dont ils sont extraits. $D$ 'emblée, l'usage fréquent d'échantillons de convenance met un doute sur la possibilité de généraliser à d'autres individus. 0 n connaît la pratique des départements de psychologie qui consiste à organiser des pools de participants qui sont plus ou moins obligés de se prêter à des recherches (Sears, 1986 ;W ells, W indschitl, 1999).

D ans les traitements, ce qui est investi par le chercheur (les traitements ou variables indépendantes) concerne à la fois le message, le contexte de communication et les caractéristiques des participants Le traitement est souvent le message lui-même (absence vs présence) ou ses propriétés syntaxiques ou sémantiques (la valence des arguments, leur force, etc.), la présence d'images plus ou moins banales ou plus ou moins choquantes, etc. Les traitements concernent encore le canal de communication (texte, vidéo, etc.) et le contexte de réception. Ici, il est courant que les chercheurs veillent à ce que les situations de communication ressemblent à celles que peut raisonnablement rencontrer un individu (validité écologique).Telle ou telle situation expérimentale rompt, plus ou moins, avec le contrat habituel de communication (biais de nouveauté du traitement ; sur la notion de contrat de communication, voir Georget, 2004). Bien évidemment, les traitements concernent les participants eux-mêmes. Pour prendre un exemple à propos d'une dimension importante pour la validité externe, le fait que les individus abordent couramment nombre de messages avec une attention minimale a été largement intégré dans les modèles récents de la persuasion (modèles fondés sur des niveaux de traitements différenciés Chaiken,Trope, 1999). Les moyens les plus divers sont utilisés :ainsi amènet-on un participant à faire deux choses en même temps (double tâche), à être confronté à des distracteurs pénalisant l'attention, ou se trouver dans une situation où ses ressources cognitives sont limitées (privation de sommeil, consommation d'alcool, etc.). Sous réserve que les résultats soient comparables, cette pluralité des indicateurs est un élément important de validité externe (fondée sur l'opérationnisme multiple ou convergent Meyer, 2002).

En matière d'observation, quels effets relève-t-on ? D'un côté, les psychologues mesurent ce qui semble les plus attestable d'un point de vue de la "réalité » externe : les jugements de valeurs (attitudes explicites), les comportements, la mémoire d'un message (reconnaissance ou rappel). D'un autre, les mesures sont plus abstraites comme les antécédents non conscients des jugements de valeur (attitudes implicites), les pensées consécutives à l'exposition à un message (élaboration cognitive), ou les indices physiologiques (rythme cardiaque, respiration, imagerie cérébrale), etc. 
Les situations dans lesquelles les données sont recueillies à un moment précis peuvent être discutées. Présenter un extrait d'un talk-show dans un laboratoire universitaire est a priori très éloigné des situations où les téléspectateurs rencontrent un talk-show chez eux, seuls, en présence de leur famille ou d'amis.Toutefois, il est possible de tester la réception d'un message en présence ou en l'absence d'un public. Rappelons que la méthode expérimentale n'implique nullement le laboratoire, et que, couramment, elle sinsère dans des terrains les plus divers. L'inscription temporelle est également décisive. II se peut que tous les participants aient partagé une histoire commune qui rend peu plausible la généralisation de leur comportement à d'autres situations que celles observées (si ce facteur, dit historique, concerne une seule modalité du traitement le problème relève de la validité interne). Une étude sur l'appréciation des risques, réalisée en décembre 2004, serait limitée du fait que tous les participants étaient exposés de manière concomitante à la médiatisation du Tsunami dans l'O céan indien.

Ces critères élémentaires (UTOS) se combinent selon une interaction qui pénalise plus ou moins la validité externe (Cook, Campbell, 1979). Considérant l'interaction entre la sélection des individus et le traitement, on peut suggérer que les participants issus d'une population particulière (par exemple, des étudiants en "infocom ») peuvent avoir une sensibilité particulière à un traitement spécifique. Si l'on sintéresse aux ressources cognitives mises en œuvre pour traiter un message publicitaire, on pourrait, par exemple, admettre que le genre " publicité " suscite chez ces derniers une attention plus grande et des connaissances plus expertes que cela n'est la cas pour d'autres populations, plus indifférentes à la publicité.

Dans le cas d'une interaction entre le site et le traitement, admettons que le propos soit de travailler sur la crédibilité de la source (scientifique vs politique). Si la recherche se passe dans un cadre universitaire, les normes portées par le site ne sont pas indifférentes à la représentation de la source du message. Et dans le cas d'une interaction entre l'histoire et le traitement, les modalités du traitement sont peut-être plus ou moins sensibles à tel ou tel contexte historique particulier. Pour reprendre l'exemple de la concomitance avec la médiatisation du Tsunami, un traitement relatif à l'état émotionnel du participant serait probablement discutable au regard de la validité externe.

\section{La réactivité de la situation expérimentale}

Un autre élément crucial de la validité externe concerne la réactivité des individus aux situations expérimentales. Concernant les effets relatifs à la séquence des traitements, dans les plans expérimentaux, il est courant 
de solliciter plusieurs fois les participants. Le fait de les questionner sur leurs croyances quant à leur capacité à interroger l'internet, avant de mesurer leurs performances, peut avoir un effet sur ces dernières (Meyer, Rodon, 2004). Solliciter les participants avant le traitement peut les amener à être sensibles à des aspects de la situation auxquels ils auraient été indifférents sans cette mesure préalable. $Q$ uand plus d'un traitement sapplique à un même participant, il est difficile de sassurer de leur indépendance. Par exemple, un traitement particulier (l'exposition à un séquence de talk-shaw) continue à être actif à l'occasion d'un autre traitement (exposition à une séquence relative aux actualités économiques) auquel le participant est exposé plus tard. Cet effet de recouvrement (ou carry-over) peut être partiellement maîtrisé par le contrôle de l'ordre de présentation des traitements.

Quant aux attentes des participants, et plus généralement les représentations des participants à propos des buts de la recherche, celles-ci peuvent être propres à une situation expérimentale particulière, et gêner fortement toute généralisation à des situations plus " naturelles " ou perçues comme telles. Le participant se fait une représentation des buts poursuivis par la recherche. Ces buts peuvent masquer les effets des traitements. Ceux des attentes de l'expérimentateur qui délivre le traitement peuvent être tout aussi importants. L'expérimentateur pourrait être plus attentionné à l'égard des participants lorsqu'il est chargé de présenter un message de prévention centré sur les pertes (conséquences négatives sur la santé) plutôt que sur les gains (avantages de la prévention). Cet effet est contrôlable par la possibilité que celui-ci ne connaisse pas les traitements qu'il affecte aux participants (procédure dite en aveugle).

\section{Tester la validité externe}

\section{Gradient de généralité : des situations aux processus}

En prime approximation, la validité externe s'apprécie au regard d'une situation, ou d'une classe de situations, à partir de laquelle on souhaite généraliser les résultats de la recherche expérimentale. Une première étape indispensable demande donc à caractériser précisément cette situation de référence. $D$ e quelle situation parle-t-on en termes d'unités (quels individus et populations $?$ ), de traitement (exposition à une ou plusieurs variables $\boldsymbol{\eta}$ ), de quels lieux (le salon familial, la salle de cinéma, la rue, le cybercafé, etc. $?$ ) et selon quelle temporalité (quelques minutes, quelques heures, plusieurs années, plusieurs dizaines d'années, etc. ?) ? Donald Campbell (1986) propose de caractériser cette comparaison systématique comme une recherche de « similarité 
proximale ", terme qu'il propose de substituer (sans postérité) à celui de validité externe. Sous cet angle, la validité externe s'exprime en gradient de généralité, depuis une situation très spécifique jusqu'à des classes générales de situation. Les critiques en termes de validité externe restent souvent à un haut degré de généralité dans le sens où l'on reproche à une recherche en laboratoire sa faible similitude avec une situation naturelle sans détailler les dimensions sur lesquelles on attend des différences (selon des critères UTO S). Ce niveau de généralisation porte donc sur les situations expérimentales elles-mêmes (voir figure 1).

Figure 1 :Deux niveaux d'appréciation de la validité externe. Le modèle (processus) et la situation expérimentale elle-même (UTOS = unités, traitements, observations et sites).

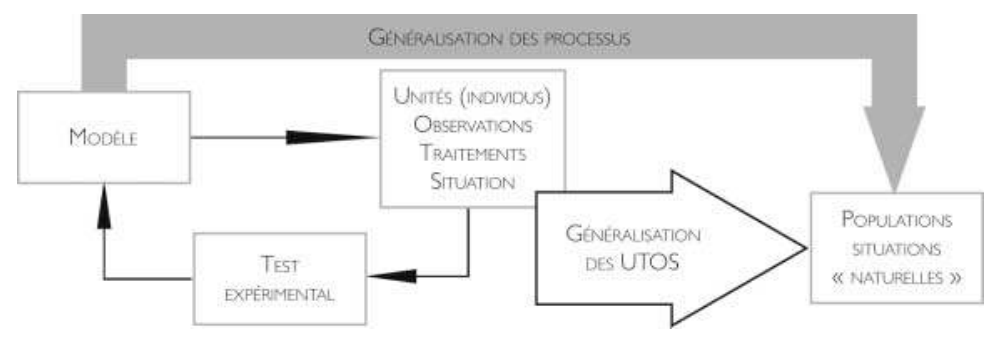

Un autre niveau est de portée beaucoup plus générale. La question est de savoir si le modèle proposé est susceptible d'être appliqué aux situations " naturelles» que l'on souhaite expliquer (voir figure 1). En psychologie, les modèles ne manquent pas, qu'ils soient de portée générale dans le domaine de cognition sociale et la persuasion (Chaiken, Trope, 1999), ou relatifs à des questions plus délimitées comme le " modèle étendu des processus parallèles " présenté par Mike Allen et Kim W itte (2004), à propos de l'effet des messages faisant « appel à la peur ". La charge de la preuve de la non-pertinence du modèle dans telle ou telle situation revient aux détracteurs du modèle, lequel repose souvent sur des programmes de recherches lourds qui ont concerné des contextes très variés. $D$ es méta-analyses ont attesté de la robustesse des effets. Ici, l'accent est mis sur les processus. Par exemple, l'argument ne porte pas sur le fait que telle expérimentation a été réalisée auprès d'étudiants, mais sur la plausibilité que les processus évoqués dans le modèle soient valables pour une autre population.

Ce niveau de généralisation - fondé sur un modèle et les processus postulés - est d'autant plus justifié qu'il est courant qu'un modèle, discuté en termes de validité externe, soit issu de situations expérimentales parfaitement non représentatives des situations 
" naturelles". De ce point de vue, il est utile de rappeler que le but d'une approche expérimentale n'est pas de reproduire plus ou moins fidèlement le réel. II s'agit de mettre à l'épreuve une hypothèse précise dans des conditions contrôlées. II se peut que ces conditions n'apparaissent jamais, sinon quand elles sont provoquées par le chercheur. La ressemblance avec des situations naturelles n'est donc pas une condition nécessaire. Sur la base de cet argument, Douglas Mook (1983) revendique la possibilité d'une « invalidité externe » pour la méthode expérimentale. Pour reprendre l'exemple initial, il est peu plausible et peu représentatif des conditions « réelles ", qu'un individu soit exposé $\mathbf{4}$ fois pendant 23 millisecondes à un visage. Toutefois, cette condition peut renseigner utilement sur le processus selon lequel la familiarité d'une source influence la valeur attribuée à un message (W eisbuch, Mackie, Garcia-Marques, 2003).

\section{Mesurer la validité externe}

Au-delà d'un cadre général de réflexion, les différences attendues entre situation expérimentale et " réalité " peuvent, bien sûr, être mises à l'épreuve par des recherches empiriques en comparant les résultats observés par d'autres méthodes. D es résultats comparables obtenus par différentes méthodes à la fois attestent de la robustesse des effets, mais donnent aussi des garanties de validité externe à la méthode expérimentale (validité dite convergente - Meyer, 2002). Un bon exemple est donné dans le domaine de l'influence des contenus médiatiques violents sur le comportement agressif (voir Girandola, 2004). D ifférentes méthodes (comparaison de groupes naturels, études longitudinales, expérimentation en laboratoire et sur le terrain) aboutissent à des effets comparables en termes de probabilité d'un effet et de taille d'effet (Anderson, Lindsay, Bushman, 2004). L'expérimentation traditionnelle peut être enrichie par d'autres méthodes. Ainsi Christina Studebacker et al. (2002) partent de l'effet de la couverture médiatique des procès sur les décisions des jurys. $\mathrm{Ce}$ domaine est travaillé sur la base de jurys simulés. L'utilisation de l'internet permet une meilleure représentativité des populations pouvant prendre part à un jury. Les défauts de la seconde approche (faiblesse du contrôle) peuvent largement être compensés par des avantages (variété accrue des populations).

L'examen systématique de la validité externe ne constitue pas une limitation seulement après-coup des résultats expérimentaux en termes de généralisation. Les critiques relatives à la validité externe sont un aiguillon indispensable qui amène à inventer de nouveaux procédés moins susceptibles de critiques. Très couramment, le chercheur anticipe les problèmes attendus et change son plan de recherche afin d'avoir des 
garanties supplémentaires en termes de validité externe si cet objectif est important pour lui. Ainsi une question classique est-elle celle de savoir si un traitement comprenant deux modalités doit être affecté à deux groupes indépendants (plan inter-sujets : deux groupes de participants sont affectés à l'une ou à l'autre des modalités) ou au même groupe de participants (lesquels sont exposés aux deux modalités). Par exemple, on peut présenter des extraits d'une campagne politique sous forme écrite ou radiophonique. Si l'objectif principal est de travailler sur le fait qu'un individu est habituellement exposé aux deux types de messages, alors le plan intra-sujet sera préféré au plan inter-sujets (Greenwald, 1976).

Pour conclure ce tour d'horizon, il faut garder à l'esprit que la validité externe a plusieurs facettes. Selon les catégories proposées par Marilynn Brewer (2000), nous avons évoqué jusqu'ici deux de ces facettes. La première est la robustesse. O n souhaite que les résultats ne résistent pas aux variations des unités, des traitements, des observations et des sites. Ce qui est à tester est le caractère répliquable de la recherche dans des contextes contrastés. De fait, c'est le moyen le plus employé pour tester la validité externe. La seconde dimension est la validité écologique :estce que les situations observées sont représentatives des situations plausibles pour les participants ? La représentativité des plans de recherche est un problème reconnu très précocement par les méthodologues. Certains traitements présentent des occurrences plus probables que d'autres, elles sont donc subjectivement plus plausibles pour l'individu. Enfin, une autre facette est la pertinence. Est-ce que, au total, la variable étudiée - quand bien même serait-elle la cause avérée de tel effet - est pertinente au regard du problème posé ? Bien évidemment, c'est du côté des recherches finalisées que ce problème se pose quand il s'agit d'aider à la décision dans une situation donnée et de faire des préconisations précises.

\section{La validité externe : un point d'entrée dans le dialogue interdisciplinaire}

Sous l'angle de la validité externe, la méthode expérimentale suscite plus de doutes que d'autres méthodes. Le souci de maximiser la validité interne qui motive le projet expérimental se paye couramment par un déficit de validité externe : «De toute évidence, aucun de ces types de validité ne le cède en importance à l'autre ; et, pourtant, il se trouve qu'ils sont relativement incompatibles entre eux : souvent, les contrôles indispensables pour assurer la validité interne tendent à en compromettre la représentativité "(Campbell, 1957 :47). Pour autant, 
la limitation du champ à quelques variables étudiées expérimentalement, en laboratoire ou en dehors du laboratoire, disqualifie-t-elle la portée pratique ou la valeur théorique des modèles ? Afin de tenter de répondre à cette question, nous avons passé en revue les voies classiques que propose la méthode expérimentale elle-même pour examiner la validité externe.

Privilégiée en psychologie, la méthode expérimentale n'a pas de vocation hégémonique. C'est un outil pragmatique qui appartient pleinement à une palette méthodologique ouverte, nécessaire pour aborder des phénomènes du niveau de complexité du champ de l'information et de la communication. À un moment ou un autre, la description libre sera utile. À un autre moment, une hypothèse sera susceptible d'être testée par voie expérimentale. II n'y pas lieu de douter que des collaborations fructueuses ne puissent sinstaurer en respectant les apports de chacun. Évidemment, cette exigence de validité externe à l'adresse de la méthode expérimentale s'applique aussi à d'autres méthodes. $A$ priori, l'enquête représentative, l'étude de cas ou l'observation approfondie sont mieux créditées en matière de validité externe.Toutefois, ce crédit repose sur une similarité de surface, plus que de fond, entre la situation étudiée et celle pour laquelle on souhaite une généralisation. Dès lors que l'on postule que les observations valent pour d'autres populations, contextes et temps, il y a lieu de regarder de plus près. Rien n'assure qu'une observation recueillie dans un contexte " naturel » donne la garantie qu'elle puisse être généralisée à un autre contexte naturel. $M$ utatis mutandis, le cadre de réflexion que nous avons développé précédemment devra s’appliquer.

\section{Conclusion}

De ce point de vue, la validité externe a une importance particulière dans un champ interdisciplinaire porté à la comparaison, voire à la confrontation des méthodologies. L'échange entre méthodes de différentes spécialités passe nécessairement par une discussion approfondie et, si possible, systématique et organisée sur la validité externe. L'examen raisonné de la validité externe est une forme opérationnelle de ce que Guy Lochard et tean-Claude Soulages (2004) désignent comme une interdisciplinarité « partagée ».

Le propos était ici volontairement limité à un point de vue de praticien des méthodes expérimentales. \& n'ai pas cherché à débattre avec les critiques épistémologiques radicales adressées à la méthode expérimentale et, plus généralement, à l'approche naturaliste ou au 
positivisme logique. Certes, on peut préférer une posture qui préserve le mystère des choses à celle qui identifie des variables pertinentes, propose des modèles explicites soumis à un test expérimental, et suscite elle-même des instruments propres à mettre en doute la portée de ses résultats.

\section{Références}

Allen M.,W itte K, 2004, « Une méta-analyse des appels à la peur :implication pour des campagnes de santé publique efficaces ", Questions de communication, 5, pp. 133-148.

Anderson C. A., Lindsay J J, Bushman B. J, 1999, « Research in the psychological Laboratory :Truth or Triviality ? ", Current Directions in Psychological Science, 8, pp. 3-9.

Aronson E, W ilson T. D., Brewer M. B., 1998, " Experimentation in social psychology ", pp. 99-142, in :Gilbert D.T., Fiske S.T., Lindzey G., eds, H andbook of social Psychology,Vol. 1, Boston, MA, McGraw-Hill.

Breckler S. J, 2004, “ Legitimate psychological Science ", M onitor on Psychology, 35, p. 22.

Brewer M. B., 2000, “ Research design and issues of validity ", pp. 17-39, in : Reis H. T., Judd C. M., eds, Handbook of Research Methods in social and personality Psychology, $\mathrm{N}$ ew York, Cambridge University Press.

Campbell D.T., 1957, « Facteurs intéressant la validité des études expérimentales dans les contextes sociaux ", pp. 47-61, in : Lemaine G., Lemaine $\mathcal{H}^{\mathrm{M}}$, dirs, Psychologie sociale et expérimentation, Paris, Mouton, 1969.

- 1986, "Relabeling internal and external validity for applied social scientists ", pp. 67-78, in :Trochim W. M. K., ed., Advances in quasi-experimental Designs and Analysis,Vol. 31, San Francisco, bssey-Bass.

Chabrol Cl., Courbet D., Fourquet-Courbet M-P, 2004, «Psychologie sociale, traitements et effets des médias ", Questions de communication, 5, pp. 5-18.

Chabrol Cl., Diligeart G., 2004, “ Préventions et risques routiers : réguler la peur et/ou la menace ? ", Questions de communication, 5, 115-132.

Chaiken S.,Trope Y.,1999, eds, Dual process Theories in social Psychology, $\mathrm{N}$ ew York, Guilford Press

CookT. D., Campbell D.T., 1979, Quasi-experimentation :Design and Analysis Issues for Field Settings, Chicago, Rand-McN ally.

Courbet D., 2004, " L'influence non consciente des publicités vues furtivement et aussitôt oubliées Une méthode d'étude socio-cognitive appliquée à linternet ", Questions de communication, 5, pp. 83-102.

Cronbach L J, 1982, Designing Evaluations of educational and social Programs, San Francisco, bssey-Bass. 
Delhomme P., Meyer T., 2001, « Mesurer les effets des campagnes de sécurité routière en Europe : quels indices et quels plans de recherche ? », Bulletin de psychologie, 54(3), 453, pp. 343-355.

- 2002, La recherche en psychologie sociale :projets, méthodes et techniques, Paris, A. Colin.

Georget P., 2004, “ Effets de stratégies publicitaires. Personnalité, contexte et attentes contractuelles ", Questions de communication, 5, pp. 69-82.

Girandola F., 2004, "Violence dans les médias: quels effets sur les comportements ", Questions de communication, 5, pp. 55-68.

Greenwald A. G., 1976, «W ithin-subjects Designs : to use or not to use », Psychological Bulletin, 83, pp. 314-320.

Lochard G., Soulages J-Cl., 2004, "Une interdisciplinarité est-elle possible ? Interrogations sur la réception des discours médiatiques ", Questions de communication, 5, pp. 19-30.

Meyer T., 2002, “ Des variables observées aux variables manipulées : un cas particulier de l'opérationnisme multiple ou convergent ", Bulletin de psychologie, 55(6), pp. 583-592.

Meyer T., Rodon C., 2004, “ Trouver sur Internet une réponse à une question ”, Hermès, 39, pp. 27-34.

Mook D. G., 1983, « In Defence of external Invalidity », American Psychologist, 38, pp. 379-387.

Radu M., 2004, « De la comparaison sociale à l'intention comportementale. Les publicités pour produits cosmétiques amincissants ", Questions de communication, 5, pp. 103-114.

Sears, D. O., 1986, « College sophomores in the laboratory :Influences of a narrow Database on social Psychology's view of human Nature ", burnal of Personality and Social Psychology, 51, 515-530.

Studebacker C.A., Robbennolt JK., Penrod S. D., Pathak-Sharma M. K., Groscup JL., Devenport J L., 2002, «Studying Pretrial Publicity Effects : New Methods for improving ecological Validity and testing external Validity ", Law and Human Behavior, 26, pp. 19-41.

W eisbuch M., Mackie D.M., Garcia-MarquesT., 2003, « Prior Source Exposure and Persuasion : further Evidence for misattributional Processes », Personality and Social Psychology Bulletin, 29, pp. 691-700. 